\title{
Visualizando os Modos Normais de Vibração com o Computador
}

\author{
S. S. B. Jácome, F. F. de Medeiros, G. Corso, e L. S. Lucena \\ International Center for Complex Systems and Departamento de Física Teórica e Experimental \\ Universidade Federal do Rio Grande do Norte, Campus Universitário \\ 59078 970, Natal, RN, Brazil
}

Recebido em 7 de março, 2002. Aceito em 3 de julho, 2002.

\begin{abstract}
Neste trabalho desenvolvemos um programa educativo versando sobre modos normais em uma cadeia de osciladores acoplados. O usuário do programa pode simular a evolução dinâmica de uma cadeia de osciladores escolhendo condições iniciais tanto em coordenadas cartesianas como em coordenadas normais. Focalizamos o efeito do desacoplamento do sistema de equações diferenciais (passagem de coordenadas cartesianas para coordenadas normais) sobre as trajetórias dos osciladores do ponto de vista da dinâmica qualitativa. Através das características da evolução dinâmica, quase-periodicidade ou periodicidade, o estudante ganha intuição sobre o significado das formas normais.
\end{abstract}

In this work we develop an educational software about normal modes in a chain of coupled oscillators. The software simulates the dynamical evolution of the system allowing to choose initial conditions in cartesian coordinates or in normal modes. We focus on the effect of decoupling the system of differential equations (transition from cartesian coordinates to normal modes) in the qualitative dynamics of the oscillators, either in the quasiperiodic or in the periodic regime. The purpose is to give the student some insights about the meaning of the normal modes.

\section{Introdução}

A utilização de programas de computador no ensino da física vem crescendo muito na última década [1-6]. Programas didáticos que auxiliam o processo de aprendizagem se encontram disponíveis ao público interessado tanto em versão comercial como gratuita. Como participantes da universidade pública, nos engajamos na tarefa de aumentar a oferta aos estudantes de materiais didáticos computacionais não pagos que venham a auxiliá-los na formação de conceitos em Física.

Diversamente da maioria dos programas de Ensino em Física que se destinam a estudantes de nível médio, o nosso é dirigido à universidade. O público-alvo são estudantes de Física, ou Engenharia, que estão cursando disciplinas de Mecânica Clássica Avançada. O objetivo do programa é facilitar ao usuário o entendimento das coordenadas normais através do jogo interativo com o computador. O programa permite que se definam as condições iniciais na forma deslocamento - velocidade bem como em coordenadas normais. Assim, o estudante pode comparar a evolução dinâmica em ambas as formas, fato que o ajuda a desenvolver o conceito e a intuição das coordenadas normais.

Nosso sistema consiste numa cadeia de $N$ os- ciladores (átomos) acoplados. Cada "átomo" se encontra a uma distância $a$ um do outro quando em repouso e está ligado por duas molas, conforme mostra a Fig. 1. As condições de contorno são fixas, isto é, as duas molas da extremidade estão presas a uma parede. Neste sistema de osciladores ligados exploraremos as coordenadas normais, também chamadas de coletivas, pois descrevem o movimento conjunto dos átomos.

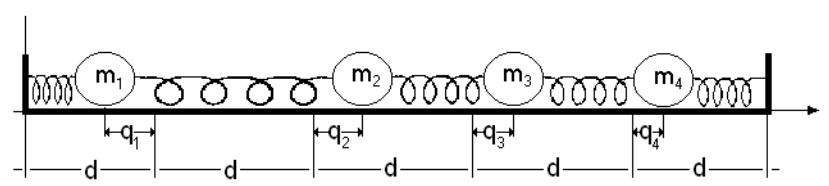

Figura 1. Esquema da cadeia de osciladores acoplados. $q_{i}$ representa o deslocamento do átomo da iésima posição .

A cadeia de osciladores é largamente estudada na Física por dois motivos, pelo menos. Primeiro, por representar um cristal monoatômico unidimensional, o mais simples dos modelos de sistemas de muitos corpos ordenados. Este modelo simplificado da rede cristalina é utilizado na estimativa do calor específico dos metais desde os primórdios da ciência do estado sólido [7]. A contagem estatística que nos fornece a energia interna de um cristal (a soma da energia das vibrações) 
é baseada nas coordenadas coletivas. Esta soma não é feita, como ingenuamente se poderia pensar, a partir das vibrações dos átomos isolados. Ademais, no estudo dos cristais, quando da passagem da Mecânica Clássica para a Mecanica Quântica, são as coordenadas normais que são quantizadas [8].

Um segundo aspecto que torna as coordenadas coletivas populares na Física Teórica é sua transição para o contínuo, isto é, o limite $a \rightarrow 0$ e $N \rightarrow \infty$. A cadeia de osciladores no limite contínuo representa o mais elementar dos sistemas mecânicos estendidos: a corda. A análise das oscilações da corda do violão, ou da viga de um edifício, tem seu modelo mais simples na versão contínua da cadeia de osciladores. E as mesmas coordenadas normais utilizadas na descrição dos sistemas discretos também são úteis na modelagem dos contínuos.

Afora as razões físicas para se estudar coordenadas normais, devemos levar em consideração o lado matemático. As coordenadas coletivas, estritamente falando, são o resultado de um desacoplamento de equações. Assim, uma solução complicada em certas coordenadas pode assumir uma forma simples em outras coordenadas. Este processo de simplificação de um problema através de uma transformação de coordenadas é comum a muitas áreas da Física. O programa que desenvolvemos tem como objetivo, também, fazer que o aluno se dê conta do quanto uma transformação de coordenadas pode simplificar a descrição de um problema.

A forma como o programa foi concebido ressalta o aspecto dinâmico dos osciladores acoplados. Conceitos como periodicidade, quase-periodicidade e caos podem surgir de uma forma espontânea e intuitiva durante a utilização do programa. De fato, sendo o sistema linear, ele não pode apresentar comportamento caótico, pois a não-linearidade é condição necessária para o aparecimento de caos em um sistema dinâmico [10]. Uma oscilação quase-periódica caracteriza-se pela combinação linear de oscilações de frequências $\Omega_{1}$ e $\Omega_{2}$ não comensuráveis entre si, $p=\frac{\Omega_{1}}{\Omega_{2}}, p \neq Q$. Isso implica que rigorosamente uma trajetória do sistema nunca voltará ao mesmo ponto. Uma observação apenas visual da evolução dinâmica de uma oscilação genérica nem sempre permite que se diferencie o caos da quase-periodicidade, enquanto uma oscilação periódica é facilmente reconhecida. O usuário do programa irá se defrontar com estas questões; efetivamente, porém, apenas duas situações são permitidas neste sistema dinâmico: quasiperiodicidade e periodicidade.

O nosso trabalho se propõe, por um lado, a ajudar o usuário a entender as coordenadas normais, e por outro, visa também estimular a prática da programação científica entre os estudantes. Nosso programa é exposto por dentro e seu algoritmo pode ser entendido com relativa facilidade. Sua utilização na construção de outros algoritmos similares, ou em seu próprio aperfeiçoamento, é encarada pelos autores como um passo a mais no processo pedagógico. Neste sentido somos construtivistas [9]. O aprendizado se dá quando o sujeito se apropria do conhecimento, usa este conhecimento, e consegue transformá-lo.

O trabalho é organizado da seguinte forma: na seção II, o modelo da cadeia de osciladores é apresentado utilizando-se da Mecânica de Newton em coordenadas convencionais (deslocamento e velocidade) bem como em coordenadas normais. As novas coordenadas facilitam a descrição e o entendimento do problema. Na seção III, o programa desenvolvido é comentado do ponto de vista de sua lógica interna. Na seção IV, a utilização do programa pelo usuário é explorada e, finalmente, na seção $\mathrm{V}$, concluímos o trabalho com uma breve avaliação pedagógica crítica.

\section{Das coordenadas cartesianas às normais}

Nesta seção apresentamos a cadeia de osciladores nas coordenadas cartesianas $q_{i}$ e depois indicaremos como se realiza a transformação que leva às coordenadas normais $Q_{i}$. No meio do caminho serão apresentadas as frequências normais, $\Omega_{i}$, prosaicamente denominadas naturais. Maiores detalhes sobre o formalismo matemático podem ser encontrados em [11, 12, 13].

Consideramos novamente a Fig. 1 e usamos a lei de Hooke para determinar a força resultante sobre o átomo da iésima posição, o que envolve o alongamento das molas adjacentes, isto é, $\left(q_{i+1}-q_{i}\right)$ e $\left(q_{i}-q_{i-1}\right)$. A segunda lei de Newton se escreve:

$$
m \ddot{q}_{i}=-k\left(q_{i+1}-q_{i}\right)+k\left(q_{i}-q_{i-1}\right) .
$$

onde os átomos têm massa $m$ e a constante de cada mola vale $k$. A condição das extremidades fixas equivale a $q_{0}=q_{N+1}=0$. Em linguagem matricial este problema assume a forma:

$$
m\left(\begin{array}{c}
\ddot{q}_{1} \\
\ddot{q}_{2} \\
\ddot{q}_{3} \\
\vdots \\
\ddot{q}_{N}
\end{array}\right)=\left(\begin{array}{ccccc}
2 k & -k & 0 & \cdots & 0 \\
-k & 2 k & -k & \cdots & 0 \\
0 & -k & 2 k & \cdots & 0 \\
\vdots & \vdots & \vdots & \ddots & \vdots \\
0 & 0 & 0 & \cdots & 2 k
\end{array}\right)\left(\begin{array}{c}
q_{1} \\
q_{2} \\
q_{3} \\
\vdots \\
q_{N}
\end{array}\right)
$$


A tarefa de encontrar as coordenadas normais do ponto de vista da álgebra linear consiste em diagonalizar o sistema (2). Elas serão as novas coordenadas do sistema diagonalizado, e as frequências normais, os elementos da diagonal principal da nova matriz. Vale a pena ressaltar que este processo só pode ser realizado se todos os autovalores do sistema forem diferentes caso contrário, a matriz canônica do sistema se encontrará na forma de Jordan [14] e não na forma diagonal.

A transformação desejada se realiza utilizando o ansatz $q_{i}=A e^{i(\Omega t \pm n \phi)}$ para resolver (1). As condições de contorno acabam por determinar as freqüências naturais:

$$
\Omega_{i}=2 \sqrt{\frac{k}{m}} \operatorname{sen}\left(\frac{\pi i}{2(N+1)}\right) .
$$

As coordenadas $q_{i}$ podem ser escritas através das coordenadas coletivas $Q_{i}$ :

$$
q_{i}=\sqrt{\frac{2}{N+1}} \sum_{j=1}^{N} \frac{1}{\sqrt{\Omega_{j}}} \operatorname{sen}\left(\frac{\pi i j}{N+1}\right) Q_{j} .
$$

A inversão deste sistema (de fato a inversão de uma matriz) permite que se explicite $Q_{i}$ em função de $q_{i}$ :

$$
Q_{i}=\sqrt{\frac{2 \Omega_{i}}{N+1}} \sum_{j=1}^{N} \operatorname{sen}\left(\frac{\pi i j}{N+1}\right) q_{j} .
$$

Retornando ao começo desta seção onde apresentamos a equação de Newton, (1), podemos agora reescrevê-la em modos normais:

$$
\frac{d^{2} Q_{i}}{d t^{2}}=-\Omega_{i}^{2} Q_{i}
$$

Esta é a grande vantagem das coordenadas normais: elas permitem o desacoplamento completo do sistema de molas. Ademais, a solução de (6) é trivial, trata-se do oscilador harmônico. Desta forma, as coordenadas normais são $N$ oscilações harmônicas cujas frequências são dadas pela equação (3). Se o sistema iniciar em uma dessas frequências, nela se manterá por tempo indeterminado. Uma condição inicial genérica, entretanto, envolve uma combinação linear de todos as coordenadas coletivas. Na próxima seção, voltaremos com todo empenho a este ponto.

\section{O Algoritmo por Dentro}

\section{III.1 Método de Euler}

Um estudante de Física passa bom tempo do seu curso estudando equações diferenciais, onde maior parte do seu estudo está dedicada a encontrar ferramentas que possam resolver tais equações . Desde então, aprende que há pelo menos dois métodos básicos que podem ser classificados como analíticos e computacionais. Comentaremos estes últimos neste artigo.
Neste artigo, escolhemos o método de integração de Euler por ser bastante eficiente, ou seja, soluções numéricas por ele encontradas condizem com a realidade do problema físico. Além disto, trata-se de um método intuitivo e de simples entendimento, o qual pode dar suporte para se entender e idealizar processos numéricos mais complicados. Uma discussão mais aprofundada sobre resolução numérica de equações diferenciais pode ser encontrada em [15].

$\mathrm{O}$ método de Euler consiste em obter, a partir de condições iniciais definidas, a solução de equações diferenciais ordinárias através da integração sucessiva das derivadas de ordem mais alta. Boas referências sobre o assunto são $[16,15]$. Ou seja, a partir da aceleração $\ddot{x}$ (segunda ordem, $\mathrm{k}=2$ ), obteremos a velocidade $\dot{x}$, (primeira ordem, $\mathrm{k}=1$ ), e então a coordenada $x$ (ordem zero, $\mathrm{k}=0$ ).

Consideremos a equação diferencial:

$$
\frac{d y}{d x}=f(x, y)
$$

onde $x$ é uma variável independente e $y$ uma variável dependente. Em muitos casos, a derivada $f(x, y)$ pode variar com o tempo (variável independente) e com a distância e velocidade, (variável dependente). Uma forma simples de se definir uma derivada é como o limite da razão entre os incrementos em $y$ e $x$ para incrementos na variável independente $t$ muito pequenos:

$$
\frac{d y}{d x}=\lim _{\Delta x \rightarrow 0} \frac{\Delta y}{\Delta x}
$$

Se conhecemos o valor de $y$ para um determinado valor $x$, podemos, então, determinar o valor da variável dependente $y$ para um valor posterior de $x$, através das definições (7) e (8). Sendo conhecidos os valores nos pontos $\left(x_{n}, y_{n}\right)$, podemos, por diferenças finitas, usando a equação (8), obter o valor aproximado de $y_{n+1}$ da seguinte forma:

$\lim _{\Delta x \rightarrow 0} \frac{\Delta y}{\Delta x}=\lim _{\Delta x \rightarrow 0} \frac{y_{n+1}-y_{n}}{x_{n+1}-x_{n}}=\lim _{h \rightarrow 0} \frac{y_{n+1}-y_{n}}{h}$

onde $h \equiv x_{n+1}-x_{n}$ é definido como passo (valor constante) de integração da equação diferencial. Se fizermos uma aproximação da eq. (7) usando a eq. (9) com valores finitos para $h$, teremos:

$$
\frac{y_{n+1}-y_{n}}{h}=f\left(x_{n}, y_{n}\right)
$$

e após algumas alterações:

$$
y_{n+1}=y_{n}+h f\left(x_{n}, y_{n}\right)
$$

que é conhecido como o Método de Integração de Euler para resolver numericamente equações diferenciais ordinárias.

Este método pode, alternativamente, ser obtido também através da Série de Taylor: 


$$
y_{n+1}=y_{n}+\left(x_{n+1}-x_{n}\right) \dot{y}\left(x_{n}, y_{n}\right)+\frac{\left(x_{n+1}-x_{n}\right)^{2}}{2} \ddot{y}\left(x_{n}, y_{n}\right)+\ldots
$$

bastando apenas truncar a série acima na primeira derivada de $y\left(x_{\mathrm{n}+1}\right)$.

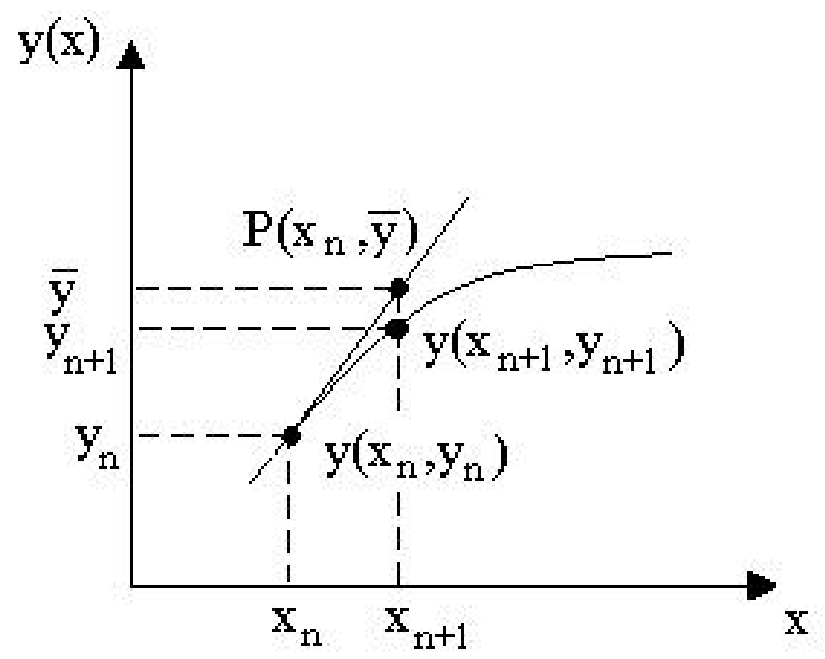

Figura 2. Esquema do integrador de Euler. O gráfico de $x$ versus $y$.

Podemos mostrar graficamente o que acontece com o processo de integração utilizando o método de Euler na Fig. 2. Observando, veremos que, se $h$ tender a zero, o ponto $Q$ se aproximará do ponto $y\left(x_{n+1}, y_{n+1}\right)$, em outras palavras, a ordenada $\bar{y}$ tenderá a $y_{n+1}$. Utilizando esse raciocínio, poderemos obter a partir do ponto $y\left(x_{n+1}, y_{n+1}\right)$, que neste caso fará o papel de valor inicial, o ponto $y\left(x_{n+2}, y_{n+2}\right)$ e assim sucessivamente até o limite do intervalo, como na Fig. 3. Note que h é pequeno o suficiente; por isso a integração computacional acompanha a curva $y(x)$.

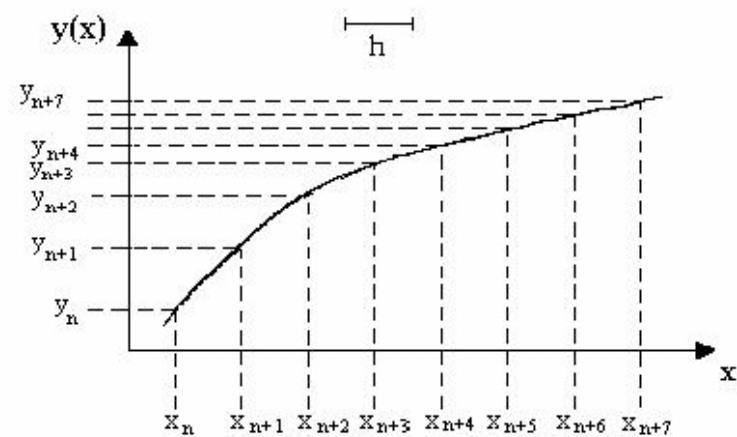

Figura 3. A partição em intervalos no integrador de Euler.

\section{III.2 Aplicação do Método de Euler}

A partir da Segunda Lei de Newton, podemos escrever de forma generalizada a aceleração $a$ em função da posição, velocidade e tempo da seguinte forma:

$$
a(x, v, t)=\frac{f(x, v, t)}{m}
$$

Sabemos, também, que a aceleração é a derivada com respeito ao tempo da velocidade, e que a velocidade é a derivada da coordenada da posição, ou seja:

$$
\begin{aligned}
& a(x, v, t)=\frac{d v}{d t} \\
& v(x, v, t)=\frac{d x}{d t}
\end{aligned}
$$

Com as equações acima, teremos condições, então, de aplicar o método de Euler para descobrir a posição e a velocidade, e com esses dados calcular a aceleração no próximo passo, desde que se saiba como $f(x, v, t)$ varia. Em forma iterativa:

$$
\begin{gathered}
a_{n}=f\left(x_{n}, v_{n}, t\right) \\
v_{n+1}=v_{n}+h a_{n} \\
x_{n+1}=x_{n}+h v_{n+1}
\end{gathered}
$$

Neste artigo, como o estudo está sendo feito sobre osciladores harmônicos simples acoplados, podemos imaginar que os mesmos são átomos, de massa $m$, ligados entre si por molas com constante $k$. Supomos também, para tornar mais fácil a implementação gráfica no computador, que o sistema tem apenas quatro átomos, sendo todas as massas e constantes de molas iguais. A função da aceleração $f$ varia apenas com a posição como na equação (1):

$$
f\left(q_{1}\right)=\frac{-k\left(q_{1+1}-q_{1}\right)+k\left(q_{i}-q_{i-1}\right)}{m} \quad i=0, . ., 4
$$

onde cada $q_{i}$ é o deslocamento da respectiva partícula $i$ em relação a sua origem.

Num algoritmo em C, o método de Euler seria basicamente:

$\mathrm{t}=0.01$

$\mathrm{q}[1]=$ valor inicial; $\mathrm{v}[1]=$ valor inicial;

$\mathrm{q}[2]=$ valor inicial; $\mathrm{v}[2]=$ valor inicial;

$\mathrm{q}[3]=$ valor inicial; $\mathrm{v}[3]=$ valor inicial;

$\mathrm{q}[4]=$ valor inicial; $\mathrm{v}[4]=$ valor inicial;

For $\left(T_{i}=0 ; T_{i} \leq T_{f} ; T_{i}+=t\right)$ 


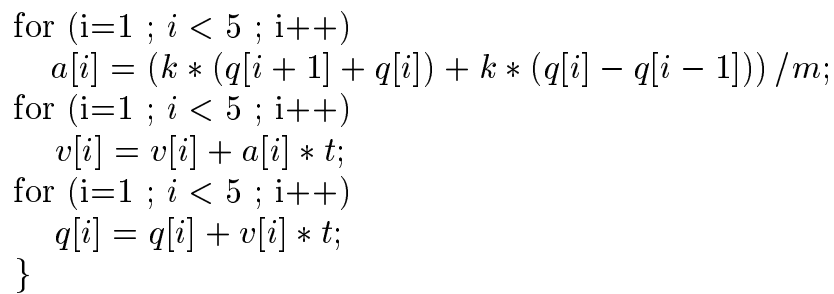

onde $T_{i}$ é o valor inicial, $T_{f}$ o valor final, e $t$ corresponde ao $h$, o incremento no método de Euler.

O algoritmo completo em $\mathrm{C}$, com seus respectivos comentários, encontra-se disponível no endereço http://www.dfte.ufrn.br/pdf.

\section{O uso Educacional do Pro- grama}

Neste capítulo descrevemos brevemente a utilização do programa, as duas janelas abertas pelo código de computador executável e as possibilidades básicas de entrada de dados no sistema. A operação do programa é simples e auto-explicativa, não necessitando qualquer treino prévio em linguagem $\mathrm{C}$, apenas uma familiaridade mínima com computadores. O programa simula a oscilatação de quatro átomos em função do tempo sendo as condições iniciais da escolha do usuário.

O programa é iniciado acionando-se seu arquivo executável, o qual abre primeiramente uma janela onde o usuário pode escolher se entrará com as posições iniciais na forma de coordenadas cartesianas ou normais. Escolhemos, como condição inicial padrão, as velocidades, em ambos os casos, iguais a zero. Isso significa que o programa dá liberdade aos átomos na rede de se deslocarem em relação à sua origem, sendo então soltos a partir do repouso.

A Fig. 4 retrata a janela principal aberta após a escolha das condições iniciais. $\mathrm{Na}$ parte superior da janela, são visíveis os quatro átomos oscilando. A trajetória de cada átomo em função do tempo está mostrada em baixo na figura. Ambas as representações são dadas em tempo real proporcionando ao usuário uma imagem realista do sistema. No canto superior, à esquerda, encontram-se os valores das condições iniciais em coordenadas normais e cartesianas.

O usuário tem duas alternativas básicas de iniciar a simulação: via modos normais, ou coordenadas cartesianas. Iniciando-se com coordenadas cartesianas genéricas, a evolução dinâmica não apresenta periodicidade visível, sendo o movimento dos átomos aparentemente descoordenado. Iniciando-se em coordenadas normais, por outro lado, a simetria do sistema é revelada, podendo facilmente a periodicidade ser observada. Condições iniciais onde apenas uma das coordenadas é diferente de zero mostram imediatamente a oscilação daquela coordenada. Este quadro pode ser observado na Fig. 4 (a) onde temos as condições iniciais em coordenadas cartesianas $q_{1}=15$ e $q_{2}=q_{3}=q_{4}=0$, e na figura em 4 (b) onde as condições iniciais são dadas em coordendas normais $Q_{1}=15$ e $Q_{2}=Q_{3}=Q_{4}=0$. Saltam aos olhos o caráter simétrico da oscilação e a periodicidade em 4 (b) contrastada com a falta de simetria e a aperiodicidade de 4 (a).

\section{Considerações Finais}

Podemos estimular nos alunos o interesse pelas disciplinas que possuem aplicações computacionais simples. No entanto, é preciso um treinamento em alguma linguagem de programação . Neste sentido, a linguagem $\mathrm{C}$ oferece ótimos recursos para implementações computacionais de simulações para a educação . O aluno, ao mesmo tempo em que se apropria do conhecimento de sala de aula ou dos livros, pode desenvolver melhor sua formação através da confecção de seus próprios programas e do uso do computador como laboratório. Como agente do aprendizado, ele passa a ser um produtor de conhecimento, e não simplesmente um receptor de informações, um agente passivo. Como o processo de construção do código computacional envolve várias etapas, entre as quais, o domínio do conteúdo teórico necessário para escrevê-lo, isso acaba estimulando no aluno o gosto pelo aprender. Dentro dessa nova visão, a participação do discente é estimulada pela criatividade. Ao educador cabe despertar a curiosidade do aluno e propor novos caminhos para o aprendizado. O computador pode se tornar, então, uma ferramenta imprescindível nesse novo contexto. Embora o uso do computador seja amplamente difundido dentro das instituições de ensino, não são todos os alunos que têm acesso a este equipamento. Alguns até mesmo alimentam uma certa aversão ao computador. Por isso, devemos buscar sempre novos caminhos para incentivar o desejo do estudante pelo aprender.

Nosso programa sobre formas normais tem como objetivo facilitar ao estudante a formação de conceitos físicos e matemáticos sobre dois sistemas de coordenadas distintos. Através da escolha das condições iniciais, o estudante interage com o computador produzindo e testando hipóteses sobre as implicações dinâmicas da escolha de coordenadas feita. Isto é, o programa permite que o estudante brinque com o computador, e, através deste jogo de exploração , estabeleça relações entre coordenadas e seu comportamento dinâmico.

Uma avaliação pedagógica do programa foi realizada com dois tipos de usuário: o iniciante que ainda não teve contato com o formalismo das formas normais, e um outro que já teve os conteúdos discutidos em aula. Mudando o tipo de entrada de dados, coordenadas cartesianas, ou normais, o aluno iniciante constrói um paradigma próprio. Através da tentativa e erro, ele desenvolveu o seguinte conceito elementar: existem coordenadas simples (onde o movimento é periódico) e coordenadas não simples (onde o movimento não apresenta periodicidade aparente). Não é claro como se 
91 $15.00\langle->\rangle$ Q1 00.40

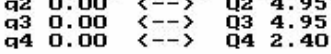

a)

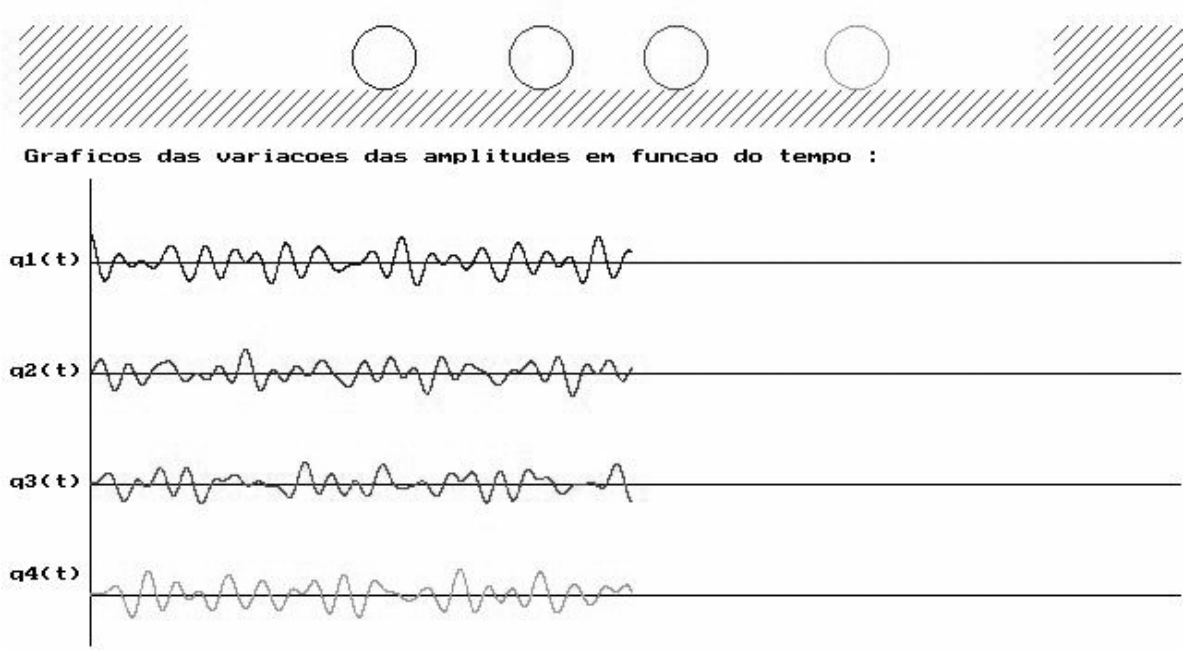

SIMULACAO DE OSCILADORES SIMPLES ACOPLADOS

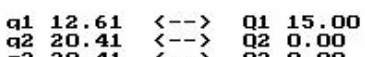

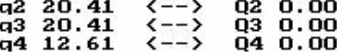

b)

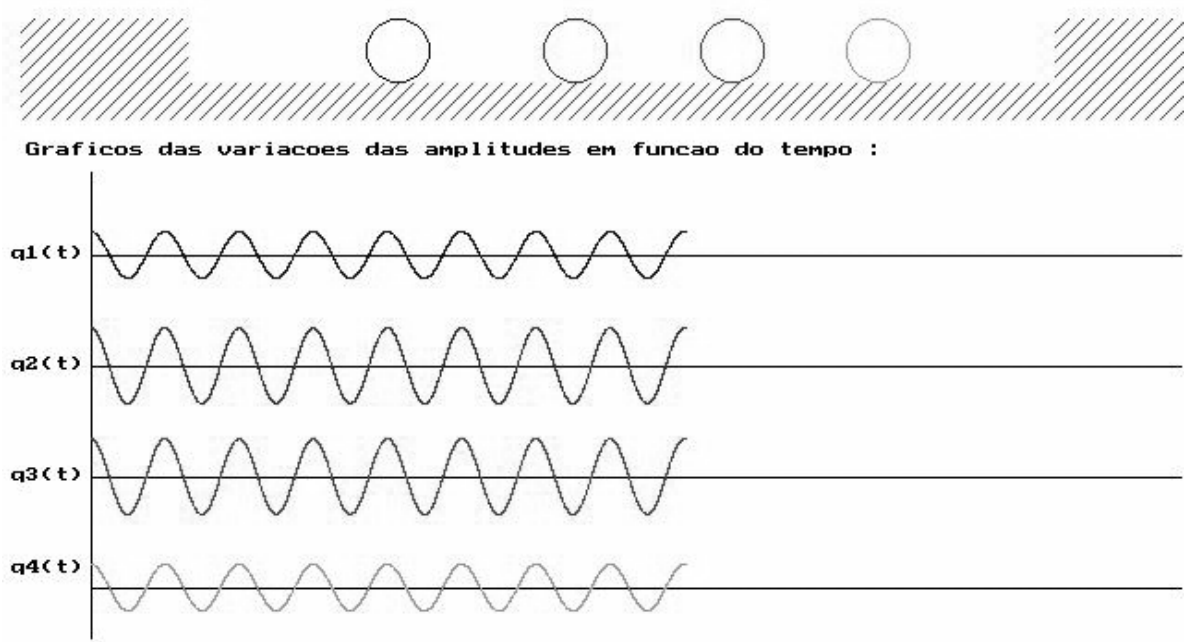

Figura 4. Uma visão da janela do computador aberta pelo programa. Na parte superior se encontra os átomos oscilando, enquanto na parte inferior a trajetória de cada átomo no tempo. Em (a) é usada a condição inicial $q_{1}=15$ e $q_{2}=q_{3}=q_{4}=0$ em coordenadas cartesianas. Em (b), a condição inicial é dada em coordenadas normais: $Q_{1}=15$ e $Q_{2}=Q_{3}=Q_{4}=0$.

O estudante que teve, anteriormente, contato com o formalismo das coordenadas normais reconheceu de imediato a implicação dinâmica dos dois tipos de coordenadas. Os conteúdos referentes à separação de variáveis ficaram mais claros e auto-evidentes. Este estudante, através do uso do computador, construiu no- vas hipóteses e consolidou os conceitos apresentados em aula.

Os pontos que mais vieram à tona durante a utilização do programa foram:

- A análise de um sistema onde todos os graus de 
liberdade estão acoplados é de difícil descrição, pois todos os $q_{i}$ (para $i \neq j$ ) influem na trajetória de um dado $q_{j}$.

- Existe um outro sistema de coordenadas onde os graus de liberdade (as vibrações) se comportam de forma periódica.

- Nas coordenadas convencionais, o movimento não apresenta periodicidade devido ao acoplamento, todos graus de liberdade interferindo com todos.

- Nas coordenadas normais, desacopladas, o movimento é simples devido à forma elementar das equações de movimento.

- As coordenadas normais representam modos coletivos de vibração que podem ser visualizados pelo computador.

- Os modos coletivos apresentam simetria, que está intrinsecamente relacionada ao fato de a vibração envolver toda a rede.

A impressão que tivemos é que o aprendizado mais significativo se deu quando o estudante teve um contato com o programa antes da aula sobre o assunto e, após ter tido os conteúdos discutidos em sala de aula, voltou às simulações. De uma forma genérica, podemos dizer que os usuários do programa avaliaram que sua compreensão da mudança de coordenadas neste problema ficou mais clara e intuitiva. Futuros usuários deste programa são convidados a nos enviarem sugestões e críticas.

\section{Agradecimentos}

Os autores agradecem à CAPES e ao CNPq o apoio financeiro a este projeto.

\section{Referências}

[1] Marisa Almeida Cavalcante, Anderson Piffer e Patrícia Nakamura, Rev. Bras. Ens. Fis. 23108 (2001).

[2] J. de S. Nogueira, C. Rinaldi, J. M. Ferreira e S. R. de Paulo, Rev. Bras. Ens. Fis. 22517 (2000).

[3] J. A. Valente, Diferentes usos do computador, Por quê o computador na Educação, http://www.proinfo.gov.br

[4] L. S. Lucena, et al., "Computadores no Ensino de Física" - Anais da 43a Reunião Anual da Sociedade Brasileira para o Progresso da Ciência, 598 (1991).

[5] L. S. Lucena et al., "Visualização do Crescimento de Polímeros em Meios Desordenados" - Anais do X Simpósio Nacional de Ensino de Física, 333 (1993).

[6] L. S. Lucena e L.R. da Silva - "Incorporação de Tópicos Atuais de Física ao Bacharelado e Licenciatura" - Anais do X Simpósio Nacional de Ensino de Física, 196 (1993).

[7] C. Kittel, Introduction to Solid State Physics (Wiley, New York, 1966).

[8] Max Born, Atomic Physics (Dover, New York, 1969).

[9] Y. de La Taille, Piaget, Vygostsky, Wallon: Teorias Psicogenéticas em Discussão (Sumus, São Paulo, 1992).

[10] E. Ott, Chaos in Dynamical Systems (Cambridge, Cambridge, 1993).

[11] G. L. Kotkin e V. G. Serbo, Problemas de Mecánica Clássica (Mir, Moscou, 1980).

[12] H. Goldstein, Classical Mechanics, (Cambridge, Mass, 1950).

[13] V. I. Arnold, Métodos Matemáticos da Mecânica Clássica (Mir, Moscou, 1987).

[14] V. I. Arnold, Equações diferenciais ordinárias (Mir, Moscou, 1984).

[15] E. Hairer, et alli Solving Differential Equations I (Springer Verlag, Series in Computational Mathematics, Vol 8, 1987).

[16] R. P. Feynman, Lectures on Physics, Vol 1, (AddisonWesley, Boston, 1963). 